\title{
A FURTHER IMPROVEMENT OF JENSEN'S INEQUALITY
}

\author{
SEVER SILVESTRU DRAGOMIR
}

\section{Introduction}

In the Theory of Inequalities, the famous Jensen's discrete inequality:

$$
f\left(\frac{1}{P_{n}} \sum_{i=1}^{n} p_{i} x_{i}\right) \leq \frac{1}{P_{n}} \sum_{i=1}^{n} p_{i} f\left(x_{i}\right)
$$

valid for every convex function $f: C \subseteq X \rightarrow \mathbb{R}$ ( $C$ is a convex subset of linear space $X)$ and for every $x_{i} \in C$ and $p_{i} \geq 0(i=1, \cdots, n)$ with $P_{n}:=\sum_{i=1}^{n} p_{i}>0$ plays such an important role that many mathematicians have tried not only to establish (1) in a variety of ways but also to find different extensions, refinements and counterparts; see [3] and [7] where further references are given.

In the recent paper [10], J. E. Pečaric and the author have obtained the following refinement of (1):

Theorem A. Let $f, x_{i}, p_{i}$ be as above and $1 \leq k \leq n$. Then one has the inequalities:

$$
\begin{aligned}
& f\left(\frac{1}{P_{n}} \sum_{i=1}^{n} p_{i} x_{i}\right) \leq \frac{1}{P_{n}^{k}} \sum_{i_{1}, \ldots, i_{k}=1}^{n} p_{i_{1}} \cdots p_{i_{k}} f\left(\frac{x_{i_{1}}+\cdots+x_{i_{k}}}{k}\right) \\
& \leq \ldots \leq \frac{1}{P_{n}^{2}} \sum_{i_{1}, i_{2}=1}^{n} p_{i_{1}} p_{i_{2}} f\left(\frac{x_{i_{1}}+x_{i_{2}}}{2}\right) \leq \frac{1}{P_{n}} \sum_{i=1}^{n} p_{i} f\left(x_{i}\right) .
\end{aligned}
$$

Another result of this type for weighted means was established by the author in [6]:

Theorem B. Let $f, x_{i}, p_{i}, k$ be as above and $q_{j} \geq 0(j=1, \cdots, k)$ with $Q_{k}:=$ $\sum_{j=1}^{k} q_{j}>0$. Then one has the inequality:

$$
f\left(\frac{1}{P_{n}} \sum_{i=1}^{n} p_{i} x_{i}\right) \leq \frac{1}{P_{n}^{k}} \sum_{i_{1}, \ldots, i_{k}=1}^{n} p_{i_{1}} \ldots p_{i_{k}} f\left(\frac{1}{Q_{k}} \sum_{j=1}^{k} q_{j} x_{i_{j}}\right)
$$

Received July 27, 1992; revised May 18, 1993. 


$$
\leq \frac{1}{P_{n}} \sum_{i=1}^{n} p_{i} f\left(x_{i}\right) .
$$

For some applications of these results in Theory of Inequalities we refer to [4], [6] and $[10]$.

The main purpose of this paper is to give an mprovement of refinement (3) as follows. Some natural applications are also pointed out.

\section{The main reults}

The following theorem holds.

Theorem. Let $f: C \subseteq X \rightarrow \mathbb{R}$ be a convex mapping on convex subset $C$ of linear space $X, x_{i} \in C, p_{i} \geq 0(i=1, \cdots, n)$ with $P_{n}>0$ and $q_{j} \geq 0(j=1, \cdots, k$; $1 \leq k \leq n$ ) with $Q_{k}>0$. Then one has the inequalities

$$
\begin{aligned}
& f\left(\frac{1}{P_{n}} \sum_{i=1}^{n} p_{i} x_{i}\right) \leq \frac{1}{P_{n}^{k}} \sum_{i_{1}, \ldots, i_{k}=1}^{n} p_{i_{1}} \ldots p_{i_{k}} f\left(\frac{x_{i_{1}}+\ldots+x_{i_{k}}}{k}\right) \\
\leq & \frac{1}{P_{n}^{k}} \sum_{i_{1}, \ldots, i_{k}=1}^{n} p_{i_{1}} \ldots p_{i_{k}} f\left(\frac{q_{1} x_{i_{1}}+\ldots+q_{k} x_{i_{k}}}{Q_{k}}\right) \leq \frac{1}{P_{n}} \sum_{i=1}^{n} p_{i} f\left(x_{i}\right) .
\end{aligned}
$$

Proof. We must prove only the second inequality.

Let consider the vectors

$$
\begin{aligned}
& y_{1}:=\frac{1}{Q_{k}}\left(q_{1} x_{i_{1}}+q_{2} x_{i_{2}}+\ldots+q_{k-1} x_{i_{k-1}}+q_{k} x_{i_{k}}\right) \\
& y_{2}:=\frac{1}{Q_{k}}\left(q_{k} x_{i_{1}}+q_{1} x_{i_{2}}+\ldots+q_{k-2} x_{i_{k-1}}+q_{k-1} x_{i_{k}}\right) \\
& y_{k-1}:=\frac{1}{Q_{k}}\left(q_{3} x_{i_{1}}+q_{4} x_{i_{2}}+\ldots+q_{1} x_{i_{k-1}}+q_{2} x_{i_{k}}\right) \\
& y_{k}:=\frac{1}{Q_{k}}\left(q_{2} x_{i_{1}}+q_{3} x_{i_{2}}+\ldots+q_{k} x_{i_{k-1}}+q_{1} x_{i_{k}}\right)
\end{aligned}
$$

where $x_{i_{j}} \in\left\{x_{1}, \ldots, x_{n}\right\}$ and $q_{j} \geq 0(j=1, \cdots, k)$ are as above.

A simple computation shows that:

$$
\frac{y_{1}+y_{2}+\ldots+y_{k-1}+y_{k}}{k}=\frac{x_{i_{1}}+x_{i_{2}}+\ldots+x_{i_{k-1}}+x_{i_{k}}}{k}
$$

and the Jensen's inequality

$$
\frac{1}{k}\left(f\left(y_{1}\right)+f\left(y_{2}\right)+\ldots+f\left(y_{k}\right)\right) \geq f\left(\frac{y_{1}+\ldots+y_{k}}{k}\right)
$$


yields that

$$
\begin{aligned}
& \frac{1}{k}\left\{f\left[\frac{1}{Q_{k}}\left(q_{1} x_{i_{1}}+q_{2} x_{i_{2}}+\ldots+q_{k-1} x_{i_{k-1}}+q_{k} x_{i_{k}}\right)\right]+\ldots\right. \\
& \left.+f\left[\frac{1}{Q_{k}}\left(q_{2} x_{i_{1}}+q_{3} x_{i_{2}}+\ldots+q_{k} x_{i_{k-1}}+q_{1} x_{i_{k}}\right)\right]\right\} \\
& \geq f\left(\frac{x_{i_{1}}+\ldots+x_{i_{k}}}{k}\right)
\end{aligned}
$$

for all $i_{1}, \ldots, i_{k}=1, \cdots, n$.

Now, if we multiply this inequality with $p_{i_{1}} \ldots p_{i_{k}} \geq 0$ and if we sum after $i_{1}, \ldots, i_{k}$ to 1 at $n$, we derive:

$$
\begin{aligned}
& \frac{1}{k}\left\{\sum_{i_{1}, \ldots, i_{k}=1}^{n} p_{i_{1}} \ldots p_{i_{k}} f\left[\frac{1}{Q_{k}}\left(q_{1} x_{i_{1}}+\ldots+q_{k} x_{i_{k}}\right)\right]+\ldots\right. \\
& \left.+\sum_{i_{1}, \ldots, i_{k}=1}^{n} p_{i_{1}} \ldots p_{i_{k}} f\left[\frac{1}{Q_{k}}\left(q_{2} x_{i_{1}}+\ldots+q_{1} x_{i_{k}}\right)\right]\right\} \\
& \geq \sum_{i_{1}, \ldots, i_{k}=1}^{n} p_{i_{1}} \ldots p_{i_{k}} f\left(\frac{x_{i_{1}}+\ldots+x_{i_{k}}}{k}\right) .
\end{aligned}
$$

Since the left membership of the above inequality is equal to

$$
\sum_{i_{1}, \ldots, i_{k}=1}^{n} p_{i_{1}} \ldots p_{i_{k}} f\left[\frac{1}{Q_{k}}\left(q_{1} x_{i_{1}}+\ldots+q_{k} x_{i_{k}}\right)\right]
$$

the proof of the theorem is finished.

Corollary 1. Let $f: C \subseteq X \rightarrow \mathbb{R}_{+}$be a convex function such that $f$ is also logarithmically concave on $C$, i.e., the mapping $\log f$ is concave on $C$. Then for all $x_{i}, p_{i}(i=1, \cdots, n), q_{j}(j=1, \cdots, k)(1 \leq k \leq n)$ as above, one has the following refinement of the arithmetic mean-geometric mean inequality:

$$
\frac{1}{P_{n}} \sum_{i=1}^{n} p_{i} f\left(x_{i}\right) \geq \frac{1}{P_{n}^{k}} \sum_{i_{1}, \ldots, i_{k}=1}^{n} p_{i_{1}} \ldots p_{i_{k}} f\left(\frac{q_{1} x_{i_{1}}+\ldots+q_{k} x_{i_{k}}}{Q_{k}}\right)
$$




$$
\begin{aligned}
& \geq \frac{1}{P_{n}^{k}} \sum_{i_{1}, \ldots, i_{k}=1}^{n} p_{i_{1}} \ldots p_{i_{k}} f\left(\frac{x_{i_{1}}+\ldots+x_{i_{k}}}{k}\right) \\
& \geq f\left(\frac{1}{P_{n}} \sum_{i=1}^{n} p_{i} x_{i}\right) \\
& \geq\left[\prod_{i_{1}, \ldots, i_{k}=1}^{n} f^{p_{i_{1}} \ldots p_{i_{k}}}\left(\frac{x_{i_{1}}+\ldots+x_{i_{k}}}{k}\right)\right]^{1 / P_{n}^{k}} \\
& \geq\left[\prod_{i_{1}, \ldots, i_{k}=1}^{n} f^{p_{i_{1}} \ldots p_{i_{k}}}\left(\frac{q_{1} x_{i_{1}}+\ldots+q_{k} x_{i_{k}}}{Q_{k}}\right)\right]^{1 / P_{n}^{k}} \\
& \geq\left[\prod_{i=1}^{n} f^{p_{i}}\left(x_{i}\right)\right]^{1 / P_{n}} .
\end{aligned}
$$

Proof. The second part of Corollary 1 follows by the above theorem for the convex mapping - $\log f$. We will omit the details.

Now, let $X$ be a real linear space and $K$ a clin in $X$, i.e., a subset of $X$ satisfying the conditions:

(KI) $\quad x, y \in \mathbb{K}$ imply $x+y \in K$;

(K2) $\quad x \in K, \alpha \geq 0$ imply $\alpha x \in K$.

Also, let suppose that $\varphi: K \rightarrow \mathbb{R}$ is a quasi-linear functional on $K$, i.e., a mapping which verifies the assumption:

$$
\varphi(\alpha x+\beta y) \leq(\geq) \alpha \varphi(x)+\beta \varphi(y)
$$

for all $\alpha, \beta \geq 0$ and $x, y \in K$. We observe that such a functional is a convex (concave) mapping on $K$ but the converse implication is not true, generally. We also observe that the following inequality holds (by induction):

$$
\varphi\left(\sum_{i=1}^{n} p_{i} x_{i}\right) \leq(\geq) \sum_{i=1}^{n} p_{i} \varphi\left(x_{i}\right)
$$

for all $p_{i} \geq 0$ and $x_{i} \in K(i=1, \ldots, n)$.

By the use of the above theorem, we can improve this inequality as follows:

Corollary 2. Let $\varphi$ be a quasi-linear functional on $K, x_{i} \in K$ and $p_{i} \geq 0$ $(i=1, \cdots, n)$ with $P_{n}>0$. Then for all $q_{j} \geq 0(j=1, \cdots, k)(1 \leq k \leq n)$ with 
$Q_{k}>0$, we have the inequalities

$$
\begin{aligned}
\sum_{i=1}^{n} p_{i} \varphi\left(x_{i}\right) & \geq(\leq) \frac{1}{Q_{k} P_{n}^{k-1}} \sum_{i_{1}, \ldots, i_{k}=1}^{n} p_{i_{1}} \ldots p_{i_{k}} \varphi\left(q_{1} x_{i_{1}}+\ldots+q_{k} x_{i_{k}}\right) \\
& \geq(\leq) \frac{1}{k P_{n}^{k-1}} \sum_{i_{1}, \ldots, i_{k}=1}^{n} p_{i_{1}} \ldots p_{i_{k}} \varphi\left(x_{i_{1}}+\ldots+x_{i_{k}}\right) \\
& \geq(\leq) \varphi\left(\sum_{i=1}^{n} p_{i} x_{i}\right) .
\end{aligned}
$$

Proof. The argument is obvious from the above theorem applied for the mapping $\varphi$.

\section{Applications}

1. Let $(X,\|\cdot\|)$ be a real normed linear space, $x_{i} \in X$ and $p_{i}, q_{j}$ are as above. Then for all $p \geq 1$ we have the following refinement of the generalized triangle inequality:

$$
\begin{aligned}
P_{n}^{p-1} \sum_{i=1}^{n} p_{i}\left\|x_{i}\right\|^{p} & \geq \frac{P_{n}^{p-k}}{Q_{k}^{p}} \sum_{i_{1}, \ldots, i_{k}=1}^{n} p_{i_{1}} \ldots p_{i_{k}}\left\|q_{1} x_{i_{1}}+\ldots+q_{k} x_{i_{k}}\right\|^{p} \\
& \geq \frac{P_{n}^{p-k}}{k^{p}} \sum_{i_{1}, \ldots, i_{k}=1}^{n} p_{i_{1}} \ldots p_{i_{k}}\left\|x_{i_{1}}+\ldots+x_{i_{k}}\right\|^{p} \\
& \geq\left\|\sum_{i=1}^{n} p_{i} x_{i}\right\|^{p} .
\end{aligned}
$$

The proof follows by the above theorem for the convex mapping $f: X \rightarrow \mathbb{R}$, $f(x):=\|x\|^{p}(p \geq 1)$.

2. Let $x_{i}>0, p_{i} \geq 0(i=1, \ldots, n)$ and $q_{j} \geq 0(j=1, \cdots, k ; 1 \leq k \leq n)$. Then the following refinement of weighted arithmetic mean-geometric mean inequality holds:

$$
\begin{aligned}
\frac{1}{P_{n}} \sum_{i=1}^{n} p_{i} x_{i} & \geq\left[\prod_{i_{1}, \ldots, i_{k}=1}^{n}\left(\frac{x_{i_{1}}+\ldots+x_{i_{k}}}{k}\right)^{p_{i_{1}} \ldots p_{i_{k}}}\right]^{1 / P_{n}^{k}} \\
& \geq\left[\prod_{i_{1}, \ldots, i_{k}=1}^{n}\left(\frac{q_{1} x_{i_{1}}+\ldots+q_{k} x_{i_{k}}}{Q_{k}}\right)^{p_{i_{1}} \ldots p_{i_{k}}}\right]^{1 / P_{n}^{k}} \\
& \geq\left(\prod_{i=1}^{n} x_{i}^{p_{i}}\right)^{1 / P_{n}}
\end{aligned}
$$

The proof follows by Corollary 1 for the mapping $f:(0, \infty) \rightarrow(0, \infty), f(x):=x$. 
3. Now, let consider the mapping $f:(0,1 / 2] \rightarrow(0, \infty)$ given by $f(x):=[x /(1-x)]^{r}$, $r \geq 1$. It is easily to see that $f$ is convex on $(0,1 / 2]$ and also logarithmically concave on this interval. By the use of Corollary 1 , one has

$$
\begin{aligned}
\frac{1}{P_{n}} \sum_{i=1}^{n} p_{i}\left(\frac{x_{i}}{1-x_{i}}\right)^{r} & \geq \frac{1}{P_{n}^{k}} \sum_{i_{1}, \ldots, i_{k}=1}^{n} p_{i_{1}} \ldots p_{i_{k}}\left(\frac{q_{1} x_{i_{1}}+\ldots+q_{k} x_{i_{k}}}{q_{1}\left(1-x_{i_{1}}\right)+\ldots+q_{k}\left(1-x_{i_{k}}\right)}\right)^{r} \\
& \geq \frac{1}{P_{n}^{k}} \sum_{i_{1}, \ldots, i_{k}=1}^{n} p_{i_{1}} \ldots p_{i_{k}}\left(\frac{x_{i_{1}}+\ldots+x_{i_{k}}}{k-x_{i_{1}}-\ldots-x_{i_{k}}}\right)^{r} \\
& \geq\left[\frac{\sum_{i=1}^{n} p_{i} x_{i}}{\sum_{i=1}^{n} p_{i}\left(1-x_{i}\right)}\right]^{r} \\
& \geq\left[\prod_{i_{1}, \ldots, i_{k}=1}^{n}\left(\frac{x_{i_{1}}+\ldots+x_{i_{k}}}{k-x_{i_{1}}-\ldots-x_{i_{k}}}\right)^{r p_{i_{1}} \ldots p_{i_{k}}}\right]^{1 / P_{n}^{k}} \\
& \geq\left[\prod_{i_{1}, \ldots, i_{k}=1}^{\left.\left(\frac{p_{1}}{q_{1}\left(1-x_{i_{1}}\right)+\ldots+q_{k}\left(1-x_{i_{k}}\right)}\right)^{r p_{i_{1}} \ldots p_{i_{k}}}\right]^{1 / P_{n}^{k}}}\right. \\
& \geq\left[\prod_{i=1}^{n}\left(\frac{x_{i}}{1-x_{i}}\right)^{r p_{i}}\right]^{1 / P_{n}} .
\end{aligned}
$$

Remark. The above inequalities contain refinements of C.-L. Wang's inequality [11] and also (if $p_{i}=1, i=1, \cdots, n$ ) of the well-known result of $\mathrm{Ky}$ Fan (see e.g. [3]):

$$
\frac{\sum_{i=1}^{n} x_{i}}{\sum_{i=1}^{n}\left(1-x_{i}\right)} \geq \prod_{i=1}^{n}\left[x_{i} /\left(1-x_{i}\right)\right]^{1 / n} .
$$

4. As in [12], we shall use the following notations:

$M=\{M \mid M$ is a positive definite matrix of order $n\}$;

$|M|=$ the determinant of the matrix $M$;

$|M|_{k}=\prod_{i=1}^{k} \lambda_{j}, k=1, \cdots, n$; where $\lambda_{1}, \ldots, \lambda_{n}$ are the eigenvalues of $M$ with $\lambda_{1} \leq \ldots \leq \lambda_{n}$, $|M|_{n}=|M|$

$M(j)=$ the submatrix of $M$ obtained by deleting the $j^{\text {th }}$ row and column of $M$;

$M[k]=$ the principal submatrix of $M$ formed by taking the first $k$ rows and columns of $M$, $M[n]=M, M[n-1]=M(n), M[0]=$ the identity matrix;

$\mathrm{BBF}=$ the class of Bellman-Bergström-Fan quasi-linear functionals $\sigma_{i}, \delta_{j}$ and $v_{k}$ defined on $M$ by

$$
\begin{aligned}
& \sigma_{i}(M):=\left|M_{i}\right|^{1 / i}, i=1, \ldots, n \\
& \delta_{j}(M):=|M| /|M(j)|, j=1, \cdots, n
\end{aligned}
$$

and

$$
v_{k}(M):=(|M| /|M[k]|)^{1 /(n-k)}, k=1, \cdots, n
$$


respectively.

It is evident that $M$ is closed under addition and multiplication by a positive number, i.e., $\mathcal{M}$ is a clin. Now, quasi-linearity of BBF-functionals follows from results in [12]:

$$
\varphi\left(p M_{1}+q M_{2}\right) \geq p \varphi\left(M_{1}\right)+q \varphi\left(M_{2}\right)
$$

for all $M_{1}, M_{2} \in M, p, q \geq 0$ and $\varphi \in \mathbb{B B F}$ (see also [3] and [8]).

In [12], C. - L. Wang has obtained the following inequality:

$$
\varphi\left(\sum_{i=1}^{m} p_{i} M_{i}\right) \geq \sum_{i=1}^{m} p_{i} \varphi\left(M_{i}\right) \geq P_{m}\left[\prod_{i=1}^{m} \varphi\left(M_{i}\right)\right]^{p_{i} / P_{m}}
$$

where $p_{i} \geq 0(i=1, \ldots, m)$, which is an interpolating inequality for

$$
\varphi\left(\frac{1}{P_{m}} \sum_{i=1}^{m} p_{i} M_{i}\right) \geq \prod_{i=1}^{m}\left[\varphi\left(M_{i}\right)\right]^{p_{i} / P_{m}} .
$$

Note that (5) is also a generalization of a result from [9].

By the use of Corollary 2, we can improve the inequality (5) as follows:

$$
\begin{aligned}
& \sum_{i=1}^{m} p_{i} \varphi\left(M_{i}\right) \leq \frac{1}{Q_{k} P_{m}^{k-1}} \sum_{i_{1}, \ldots, i_{k}=1}^{m} p_{i_{1}} \ldots p_{i_{k}} \varphi\left(q_{1} M_{i_{1}}+\ldots+q_{k} M_{i_{k}}\right) \\
\leq & \frac{1}{k P_{m}^{k-1}} \sum_{i_{1}, \ldots, i_{k}=1}^{m} p_{i_{1}} \ldots p_{i_{k}} \varphi\left(M_{i_{1}}+\ldots+M_{i_{k}}\right) \\
\leq & \varphi\left(\sum_{i=1}^{m} p_{i} M_{i}\right)
\end{aligned}
$$

where $1 \leq k \leq m, M_{i} \in \mathcal{M}, \varphi \in \mathrm{BBF}, p_{i} \geq 0(i=1, \ldots, m)$ with $P_{m}>0$ and $q_{j} \geq 0$ $(j=1, \cdots, k ; 1 \leq k \leq m)$ with $Q_{k}>0$.

\section{References}

[1] H. A.lzer, "A. converse of Ky Fan's inequalities", C. R. Math. Rep. Acad. Sci. Canada, 9 (1989), 1-3.

[2] R. E. Barlow, A. W. Marschal and F. Proschan, "Some inequalities for starshaped and convex functions", Pacific J. Math., 19 (1969), 19-42.

[3] E. F. Beckenbach and R. Bellman, "Inequalities", 4-th ed., Springer Verlag, Berlin, 1983.

[4] S. S. Dragomir, "A refinement of Jensen inequality", G. M. Metod. (Bucharest), 10 (1989), 190-191.

[5] S. S. Dragomir and N. M. Ionescu, "On some inequalities for convexdominated functions", Anal. Num. Theor. Approx., 19 (1990), 21-28.

[6] S. S. Dragomir, "Some refinements of Ky Fan's inequality", J. Math. Anal. Appl. 163 (1992), 317-321.

[7] D. S. Mitrinović, "Analytic Inequalities", Springer Verlag, Berlin, 1970. 
[8] D. S. Mitrinović and J. E. Pečarić, "Determinantal inequalities of Jensen'a type", Ann. Oster. Akad. Wiss. Math. Klasse, 125 (1988), 75-78.

[9] L. Mirsky, "An inequality for positive definite matrices", Amer. Math. Montly, 62 (1955), 428-430.

[10] J. E. Pečarić and S. S. Dragomir, "A refinement of Jensen inequality and applications", Studia Math. Univ. "Babes-Bolyai", 34 (1) (1989), 15-19.

[11] C.-L. Wang, "An a Ky Fan inequality of the complementary A.-G. type and its variants", J. Math. Anol. Appl., 73 (1980), 501-505.

[12] C.-L. Wang, "Extensions of determinantal inequalities", Utilitas Math., 13 (1978), 201-210.

Department of Mathematics, University of Timişoara, B-dul V. Pârvan 4, R-1900 Timişoara, România. 